\title{
Effective Feature Selection for Classification of Malware Families
}

\author{
Taeyoung Jeong ${ }^{1}$, Sungwon $\mathrm{Lee}^{2}$, Jonghee Youn ${ }^{3}$, Doosan $\mathrm{Cho}^{4}$ \\ ${ }^{I}$ Student, Dept. of Computer Engineering, Yeungnam Univ., 280 Daehak-Ro, Gyeongsan, Gyeongbuk, \\ Republic of Korea, somuch07@naver.com \\ ${ }^{2}$ Student, Dept. of Computer Engineering, Yeungnam Univ., 280 Daehak-Ro, Gyeongsan, Gyeongbuk, \\ Republic of Korea,noke15@ynu.ac.kr \\ ${ }^{3}$ Professor, Dept. of Computer Engineering, Yeungnam Univ., 280 Daehak-Ro, Gyeongsan, \\ Gyeongbuk, Republic of Korea, youn@yu.ac.kr \\ ${ }^{4}$ Professor, Dept. of Electrical \& Electronic Engineering, Sunchon National University, Suncheon, \\ Jeollanam-do, Republic of Korea, dscho@scnu.ac.kr
}

Corresponding author: Jonghee Youn

\begin{abstract}
Nowadays, numerous new malwares are produced and distributed every day. Modified or new types of malware, which are different from existing malware, have been identified. It is very inefficient for analysts to manually analyze those types of malware one by one from the beginning. This paper presents a method to utilize API and DLL call frequency to detect and classify malware families efficiently. Those APIs and DLLs that are related to malware are selected, the calling patterns that appear in the process of actually analyzing the APIs and DLLs are quantified, and the results are used as a malware classification filter. An automatic analysis system is constructed to increase the efficiency of analysis of a large number of pieces of malware. Then, the features of each malware family are extracted using the results of analysis for malware classification. Data mining and open source tools are used to enhance the efficiency and accuracy of analysis. Based on the result of analyzing actual malware, it was found that it is sufficiently effective to detect and classify malicious codes through feature selection using APIs and DLLs. This paper proposes a new criterion for detecting and classifying malicious codes, and can be applied to or referred to other malicious code research in the future to improve the accuracy and efficiency of analysis.
\end{abstract}

Keywords: Malware Analysis, Automatic Analysis System, Malware Family Classification, Malware Detection

\section{Introduction}

Computers and the Internet have already become widely available from a long time ago. Nowadays, diverse functions have been developed for the convenience of users to the extent that nothing is impossible with computers. A problem is that as computers are used in diverse systems and methods, the breadth of personal information used has been widened, and cybercrimes targeting the personal information have increased[1][2]. Distributing malware that runs on computers to perform malicious actions is one of the methods used in cybercrimes. Studies of attacking methods, detection methods to prevent attacks, classification methods, etc. have been importantly carried out in the field of security from the past and are still actively in progress.

Received: September 23, 2020; 1st Review Result: November 12, 2020; 2nd Review Result: December 28, 2020 Accepted: January 25, 2021 
Methods to efficiently analyze malware for security studies include an automated analysis system[3]. Since the number of pieces of malware is very large and the types of malware are much diverse, it is time-consuming and very inefficient for analysts to manually analyze them individually. The features of malware or standards that will enable classification may be determined, and an automated analysis system may be constructed to efficiently analyze malware according to the importance or purposes of malware. However, in recent years, the types of malware have been diversified, and those types of malware that are variants of the existing well-known malware and become new threats have been increasing while those types that can be hardly detected with existing systems have been discovered. Malware is also technically advancing as attackers hide malware through obfuscation or design malware more complex to prevent the malware from being analyzed[4].

In this paper, malware is analyzed and a new type of automated analysis system is presented. The analysis system targets those pieces of malware that run on Windows systems, and is designed with the frequency of calls of API and DLL as its core content. Instead of being designed from scratch, the analysis system is designed using Cuckoo Sandbox[5] and WEKA[6], which are existing open source tools. Cuckoo Sandbox is used to dynamically analyze malware in independent systems, and the results are quantified through WEKA, which is a data mining tool. An analysis method is presented from a perspective different from the existing one to solve problems, and portability to other related studies is improved through the use of open source tools with a view to providing an efficient system capable of secondary processing of data in accordance with changing pieces of malware.

\section{Related Works}

Studies related to family classification through automatic malware analysis and feature extraction and combination are diverse. Among them, the cores and features of the contents of the studies referred to in the present study are briefly summarized.

There is a study titled 'An Approach for Detection and Family Classification of Malware Based on Behavioral Analysis' [7] conducted by Aalborg University. In this study, malware was automatically analyzed dynamically using Cuckoo Sandbox. The analysis results were applied to machine learning to detect malware and classify families. More than 270,000 malware samples and 837 cleanware samples were used for the study. A total of five features were selected for family classification: API Sequence, API Frequency, API Bins, DLL Frequency, and Signature. With these features, malware is classified into 5 families: Small, OnlineGames, Hupigon, Frethog, and Zlob.

A study titled Malware Family Recommendation using Multiple Sequence Alignment[8] is the content of a paper published in 2015. This paper applies Multiple Sequence Alignment[9] to detect and classify variant malware. This paper identifies the information on the APIs called from malware and lists the names of the APIs called in order of call to form an API call sequence. New malware families are classified by comparing the similarity of their API sequences among malware families classified in the existing method.

The paper titled 'Evolutionary Algorithms for Classification of Malware Families through Different Network Behaviors' [10] was published by M. Zubair Rafique, Ping Chen, Christophe Huygens, and Wouter Joosend. Unlike previous papers, this paper introduces a method of classifying malware families through network communication analysis. Similar patterns appear in network requests created by malware when malware communicates with a server. Malware is classified through these features. Although it is not easy to identify patterns because various types of protocols can be used, it can be a good family classification criterion in that it is not affected by code obfuscation, etc.

Finally, the study titled "Feature Selection and Extraction for Malware Classification"[11] conducted by the Department of Electrical Engineering National Taiwan University of Science and Technology proposes a general and efficient algorithm for classifying malware. This study was conducted in 5 steps 
based on malware behaviors collected in the Sandbox environment, and was conducted on actual data sets with 4288 samples of 9 families.

\section{Proposed Techniques}

This section describes a study conducted to determine features based on appropriate combinations of the API category frequency, API call frequency, and DLL frequency out of the results obtained by dynamically analyzing malware using Cuckoo Sandbox. The dynamic analysis of malware was carried out using the functions basically supported by Cuckoo Sandbox, then, collected and processed API or DLL call information from the results organized after dynamic analysis. API categories were divided referring to An Approach for Detection and Family Classification of Malware Based on Behavioral Analysis[7]. [Table 1] is a list of the classified API categories with related functions.

The categories shown in [Table 1] are groups of highly related APIs that are frequently called from malware. The entire 157 APIs were divided into 13 categories. [Table 2] is a list of process related APIs belonging to the category Process among the categories. As shown in [Table 2], the categories are efficiently used for family classification because each category groups many related API lists into one.

[Table 1] API Category List with Functions

\begin{tabular}{|c|c|}
\hline Category Name & Functions \\
\hline 1. Device & DeviceIoControl, NtDeviceloControlFile \\
\hline 2. Filesystem & $\begin{array}{l}\text { CopyFileA, CopyFileExW, CopyFileW, CreateDirectoryExW, CreateDirectoryW, } \\
\text { DeleteFileW, DeleteFileA, FindFirstFileExA, FindFirstFileExW, } \\
\text { MoveFileWithProgressW, RemoveDirectoryA, RemoveDirectoryW, NtCreateFile, } \\
\text { NtDeleteFile, NtOpenDirectoryObject, NtOpenFile, NtQueryDirectoryFile, } \\
\text { NtQueryInformationFile, NtReadFile,NtSetInformationFile, NtWriteFile, } \\
\text { NtCreateNamedPipeFile }\end{array}$ \\
\hline 3. Misc & WriteConsoleA, WriteConsoleW, GetCursorPos, GetSystemMetrics \\
\hline 4. Process & $\begin{array}{l}\text { CreateProcessInternalW, ReadProcessMemory, WriteProcessMemory, system } \\
\text { NtCreateProcess, NtCreateSection, NtCreateUserProcess, NtFreeVirtualMemory, } \\
\text { NtMakeTemporaryObject, NtOpenSection, NtProtectVirtualMemory, } \\
\text { NtMakeTemporaryObject, NtOpenSection, NtProtectVirtualMemory, } \\
\text { NtReadVirtualMemory, NtTerminateProcess, NtWriteVirtualMemory, } \\
\text { ShellExecuteExW, VirtualProtectEx, ExitProcess, ZwMapViewOfSection }\end{array}$ \\
\hline 5. Registry & $\begin{array}{l}\text { NtCreateProcess, NtCreateSection, NtCreateUserProcess, NtFreeVirtualMemory, } \\
\text { NtMakeTemporaryObject, NtOpenSection, NtProtectVirtualMemory, } \\
\text { NtMakeTemporaryObject, NtOpenSection, NtProtectVirtualMemory, } \\
\text { NtReadVirtualMemory, NtTerminateProcess, NtWriteVirtualMemory, } \\
\text { ShellExecuteExW, VirtualProtectEx, ExitProcess, ZwMapViewOfSection, NtOpenKey, } \\
\text { NtOpenKeyEx, NtQueryKey, NtQueryMultipleValueKey, NtQueryValueKey, } \\
\text { NtSaveKeyEx, NtSaveKey, NtSetValueKey }\end{array}$ \\
\hline 6. Services & $\begin{array}{l}\text { ControlService, CreateServiceA, CreateServiceW, DeleteService, OpenSCManagerW, } \\
\text { OpenSCManagerA, OpenServiceA, OpenServiceW, StartServiceW, StartServiceA }\end{array}$ \\
\hline 7. Socket & $\begin{array}{l}\text { WSARecv, WSARecvFrom, WSASend, WSASendTo, WSASocketA, WSASocketW, } \\
\text { WSAStartup, socket, ioctlsocket, closesocket, setsockopt }\end{array}$ \\
\hline 8. Synchronization & Synchronization, NtCreateMutant, NtOpenMutant, NtDelayExecution \\
\hline 9. System & $\begin{array}{l}\text { LookupPrivilegeValueW, LdrGetDllHandle, LdrGetProcedureAddress, LdrLoadDll, } \\
\text { ExitWindowsEx }\end{array}$ \\
\hline 10. Threading & $\begin{array}{l}\text { ExitThread, CreateRemoteThread, CreateThread, NtCreateThreadEx, } \\
\text { NtGetContextThread, NtOpenThread, NtResumeThread, NtSetContextThread, }\end{array}$ \\
\hline
\end{tabular}




\begin{tabular}{|c|l|}
\hline & NtSuspendThread, NtTerminateThread, RtlCreateUserThread \\
\hline & $\begin{array}{l}\text { DnsQuery_A, URLDownloadToFileW, HttpOpenRequestA, HttpOpenRequestW, } \\
\text { HttpSendRequestA, HttpSendRequestW, InternetCloseHandle, InternetConnectW, } \\
\text { InternetConnectA, InternetOpenA, InternetOpenW, InternetOpenUrlA, } \\
\text { InternetOpenUrlW, InternetReadFile, InternetWriteFile, GetAddrInfoW, TransmitFile, } \\
\text { accept, bind, connect, getaddrinfo, gethostbyname, listen, recv, recvfrom, select, send, } \\
\text { sendto, shutdown }\end{array}$ \\
\hline 11. Network & FindWindowW, FindWindowA, FindWindowExW, FindWindowExA \\
\hline 13. Windows & SetWindowsHookExA, SetWindowsHookExW, UnhookWindowsHookEx \\
\hline
\end{tabular}

[Table 2] Functions that Belong to the Category Process

\begin{tabular}{|c|c|}
\hline Functions & Description \\
\hline CreateProcessInternalW & Creates a new process and its primary thread. \\
\hline ReadProcessMemory & $\begin{array}{l}\text { Copies the data in the specified address range from the address space of the } \\
\text { specified process into the specified buffer of the current process. }\end{array}$ \\
\hline WriteProcessMemory & $\begin{array}{l}\text { Copies the data from the specified buffer in the current process to the address range } \\
\text { of the specified process. }\end{array}$ \\
\hline System & Executes an internal operating system command. \\
\hline NtCreateProcess & Create a process. \\
\hline NtCreateSection & $\begin{array}{l}\text { Create a section object and opens a handle to the object with the specified desired } \\
\text { access. }\end{array}$ \\
\hline NtFreeVirtualMemory & Deletes a region of pages within the virtual address space of a subject process. \\
\hline NtMakeTemporaryObject & Clears object's PERMANENT flag. \\
\hline NtOpenSection & Opens a handle to a section object with the specified desired access. \\
\hline NtReadVirtualMemory & $\begin{array}{l}\text { Copies the specified address range from the specified process into the specified } \\
\text { address range of the current process. }\end{array}$ \\
\hline NtTerminateProcess & Kills the process \\
\hline NtWriteVirtualMemory & $\begin{array}{l}\text { Copies the specified address range from the current process into the specified } \\
\text { address range of the specified process. }\end{array}$ \\
\hline ShellExecuteExW & Performs an operation on a specified file. \\
\hline VirualProtectEx & $\begin{array}{l}\text { Changes the protection on a region of committed pages in the virtual address space } \\
\text { of a specified process. }\end{array}$ \\
\hline ExitProcess & Ends the calling process and all its threads. \\
\hline
\end{tabular}

When an API in Table 1 has been called among the APIs identified when malware is run, the frequency of calling by category are counted. The categories are represented by numbers and saved as Json files for later use as a feature for family classification. The contents being saved include not only the frequency of calling counted for the categories of APIs grouped, but also the frequency of calling counted for each of the 157 APIs.

The frequency of DLL calls are measured. The DLLs frequently used in malware are numbered as with APIs and the frequency of calls are counted when the DLLs are called. [Table 3] shows 25 
commonly used DLLs and the numbering. The frequency of DLL calls are also saved as a Json files as data for family classification.

[Table 3] DLL Call Frequency Criteria

\begin{tabular}{|c|c|c|}
\hline 1. kernelbase.dll & 10. crytbase.dll & 19. ntdll.dll \\
\hline 2. user32.dll & 11. rrcrt4.dll & 20. rpcrt4.dll \\
\hline 3. mscoree.dll & 12. urlmon.dll & 21. setupapi.dll \\
\hline 4. advapi32.dll & 13. uxtheme.dll & 22. propsys.dll \\
\hline 5. kernel32.dll & 14. dwmapi.dll & 23. apphelp.dll \\
\hline 6. ole32.dll & 15. wininet.dll & 24.api-ms-win-security \\
& & -sddl-11-1-0.dll \\
\hline 7. ole32aut.dll & 16. gdi32.dll & 25. rpcss.dll \\
\hline 8. comctl32.dll & 17. profapi.dll & \\
\hline 9. shell32.dll & 18. shlwapi.dll & \\
\hline
\end{tabular}

When the series of processes has been undergone, three feature data can be collected: API category frequency, API call frequency, and DLL call frequency. WEKA, which is an open source tool, is used to carry out data mining with the generated feature datasets to check whether the datasets are valid. WEKA is a data mining tool developed by Waikato University in New Zealand and supports data preprocessing, feature selection, clustering, visualization, classification, and regression analysis. Among the functions of WEKA, the 'Explorer' function can be used to analyze a certain data set to be analyzed by applying diverse algorithms. [Fig. 1] is a flow chart that summarizes the entire procedure of the proposed method. After analysis through Cuckoo Sandbox, check API and DLL information. Data mining the information with the WEKA tool to check the features of the malware family.

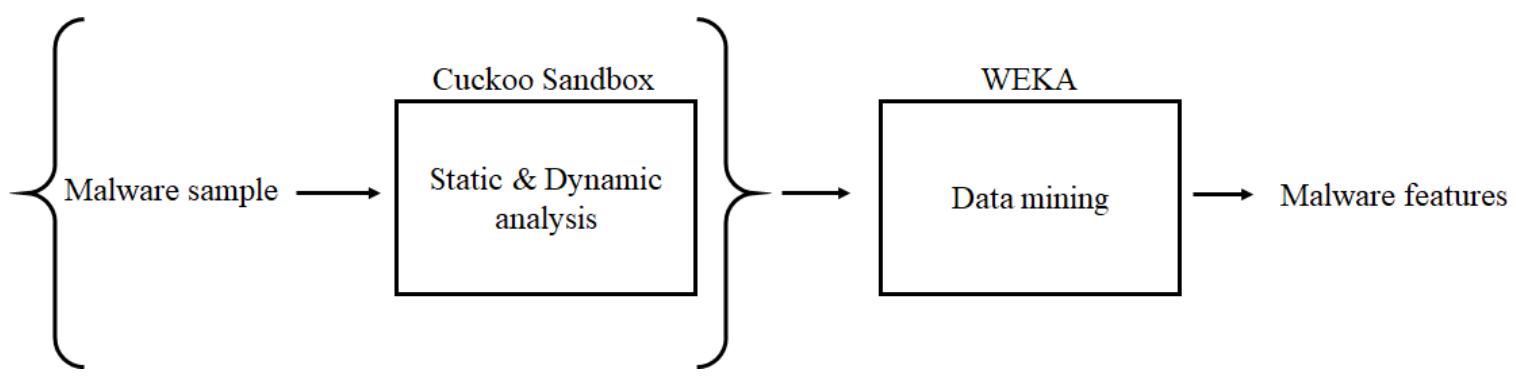

[Fig. 1] The Overall Flow of the Proposal Method

\section{Experimental Result}

A total of 148 classified sample data were applied to WEKA and were classified into five families: Small, OnlineGames, Hupigon, Frethog, and Zlob. The classification of the relevant malware was based on Virustotal's analysis report. Those pieces of malware that had no classification results were classified as NONE. [Fig. 2] is the screen of the result of running WEKA by applying API category frequency, API call frequency, and DLL call frequency. 


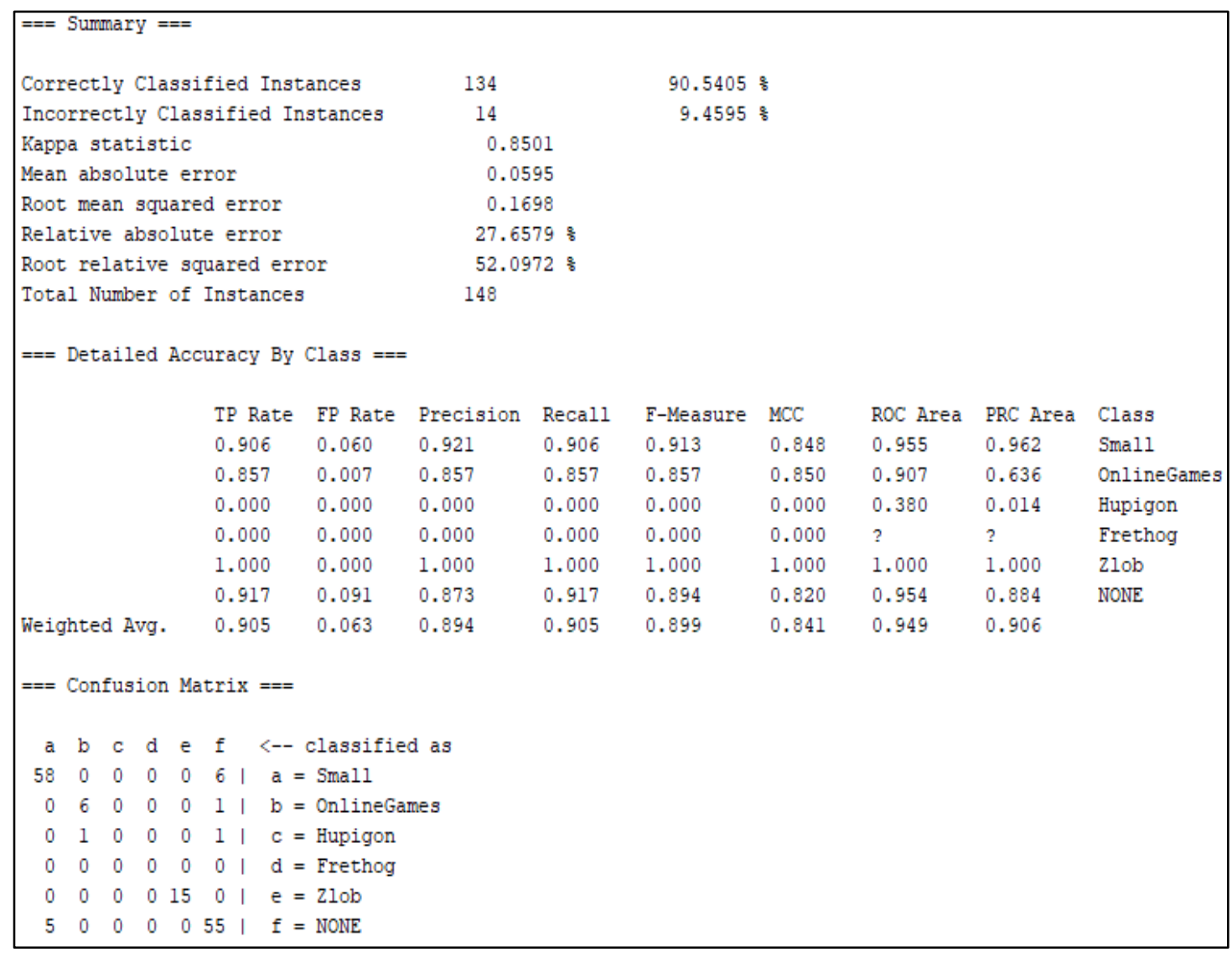

[Fig. 2] Result of WEKA Applied with Three Features

The 148 pieces of malware were classified into 7 for OnlineGames, 2 for Hupigon, 15 for Zlob, 0 for Frethog, and 60 for other NONE data. The number of instances accurately classified was found to be 134 , which is $90.54 \%$, and the number of instances not accurately classified was found to be 14 , which is $9.46 \%$ as shown in Table 4. In addition, values such as the True Positive (TP) Rate, False Positive (FP) Rate, and precision are shown by family, and are also shown in the form of confusion matrices. The TP Rate refers to the rate of those pieces of malware that correspond to A to be classified into A, and the FP Rate refers to the rate of those pieces of malware that do not correspond to A to be classified into A. Precision refers to the ratio of those pieces of malware that actually correspond to A to those pieces of malware that were classified into A. The TP Rates and the precision closer to 1 show better performance and the FP Rates closer to 0 show better performance. In the case of Hupigon and Frethog, the detailed values were 0 in many cases because the numbers of samples were small or 0 . Since the number of samples were small and the distributions were uneven, the statistics of diverse combinations of features are checked rather than the statistics by sample.

[Table 4] Result of Classification with All Three Features

\begin{tabular}{|l|c|c|c|}
\hline \multicolumn{1}{|c|}{ Class } & TP & FP & Pre \\
\hline Small & 0.906 & 0.060 & 0.921 \\
\hline OnlineGames & 0.857 & 0.007 & 0.857 \\
\hline Hupigon & 0.000 & 0.000 & 0.000 \\
\hline Frethog & 0.000 & 0.000 & 0.000 \\
\hline Zlob & 1.000 & 0.000 & 1.000 \\
\hline None & 0.917 & 0.091 & 0.873 \\
\hline Average & 0.905 & 0.063 & 0.894 \\
\hline
\end{tabular}


Only one of the three features was applied at a time and the results were identified. First, the feature API category classification was applied to run WEKA and the results were identified. [Table 5] shows the result of classification. The number of instances accurately classified was 127 , which was $85.81 \%$, and the number of instances not accurately classified was 21 , which was $14.19 \%$. In addition, the mean TP Rate, FP Rate, and Precision were shown to be $0.858,0.085$, and 0.847 , respectively, which were generally lower than the results of the combination of all three features.

[Table 5] Result of Classification (only API category)

\begin{tabular}{|l|c|c|c|}
\hline \multicolumn{1}{|c|}{ Class } & TP & FP & Pre \\
\hline Small & 0.844 & 0.083 & 0.885 \\
\hline OnlineGames & 0.857 & 0.007 & 0.857 \\
\hline Hupigon & 0.000 & 0.000 & 0.000 \\
\hline Frethog & 0.000 & 0.000 & 0.000 \\
\hline Zlob & 0.800 & 0.023 & 0.800 \\
\hline None & 0.917 & 0.114 & 0.846 \\
\hline Average & 0.858 & 0.085 & 0.847 \\
\hline
\end{tabular}

[Table 6] Result of Classification (only API Call Frequency)

\begin{tabular}{|l|c|c|c|}
\hline \multicolumn{1}{|c|}{ Class } & TP & FP & Pre \\
\hline Small & 0.906 & 0.071 & 0.906 \\
\hline OnlineGames & 0.857 & 0.007 & 0.857 \\
\hline Hupigon & 0.000 & 0.000 & 0.000 \\
\hline Frethog & 0.000 & 0.000 & 0.000 \\
\hline Zlob & 1.000 & 0.015 & 0.882 \\
\hline None & 0.900 & 0.068 & 0.900 \\
\hline Average & 0.899 & 0.060 & 0.887 \\
\hline
\end{tabular}

Next, only the feature API call frequency was applied to run WEKA and the results were identified as shown in [Table 6]. The number of instances accurately classified was 133 , which is $89.9 \%$, and the number of instances not accurately classified was 15 , which was $10.1 \%$. In addition, the mean TP Rate, FP Rate, and Precision were shown to be $0.899,0.060$, and 0.887 , respectively, which were generally very minutely lower compared to the result of the combination of all three features. It can be seen that the performance was slightly improved compared to the results when only the feature category frequency was applied. Thereafter, only the feature DLL call frequency was applied to run WEKA and the results were identified. In [Table 7], the number of instances accurately classified was 122 , which is $82.4 \%$, and the number of instances not accurately classified was 26 , which was $17.6 \%$. In addition, the mean TP Rate, FP Rate, and Precision were shown to be 0.824, 0.112, AND 0.817, respectively, which were generally much lower compared to when the combination of all three features was applied, when only the feature API category was applied, or when only the feature API frequency was applied.

[Table 7] Result of Classification (only DLL Call Frequency)

\begin{tabular}{|l|c|c|c|}
\hline \multicolumn{1}{|c|}{ Class } & TP & FP & Pre \\
\hline Small & 0.891 & 0.036 & 0.950 \\
\hline OnlineGames & 0.857 & 0.007 & 0.857 \\
\hline Hupigon & 0.000 & 0.000 & 0.000 \\
\hline Frethog & 0.000 & 0.000 & 0.000 \\
\hline
\end{tabular}




\begin{tabular}{|l|l|l|l|}
\hline Zlob & 1.000 & 0.015 & 0.882 \\
\hline None & 0.950 & 0.102 & 0.864 \\
\hline Average & 0.912 & 0.057 & 0.903 \\
\hline
\end{tabular}

Thereafter, the combinations of two out of the three features were applied and the results were identified. When the API frequency and DLL frequency were used as features, the number of instances accurately classified was 135 , which is $91.2 \%$, and the number of instances not accurately classified was 13, which was $8.78 \%$. In addition, the mean TP Rate, FP Rate, and Precision were shown to be 0.912 , 0.057 , and 0.903 , respectively, which were the most excellent results among the results of experiemts conducted. When the API category frequency and DLL frequency were used as features, the number of instances accurately classified was 131 , which was $88.5 \%$, and the number of instances not accurately classified was 17 , which was $11.5 \%$. In addition, the mean TP Rate, FP Rate, and Precision were shown to be $0.885,0.077$, and 0.876 , respectively, which were slightly worse than the results when all three features were used. When the API category frequency and API frequency were used as features, the number of instances accurately classified was 134 , which was $90.5 \%$, and the number of instances not accurately classified was 14 , which was $9.5 \%$. In addition, the mean TP Rate, FP Rate, and Precision were shown to be $0.905,0.060$, and 0.893 , which were very similar to the results when all three features were used with quite minute differences. The contents of analysis are summarized in [Fig 3].

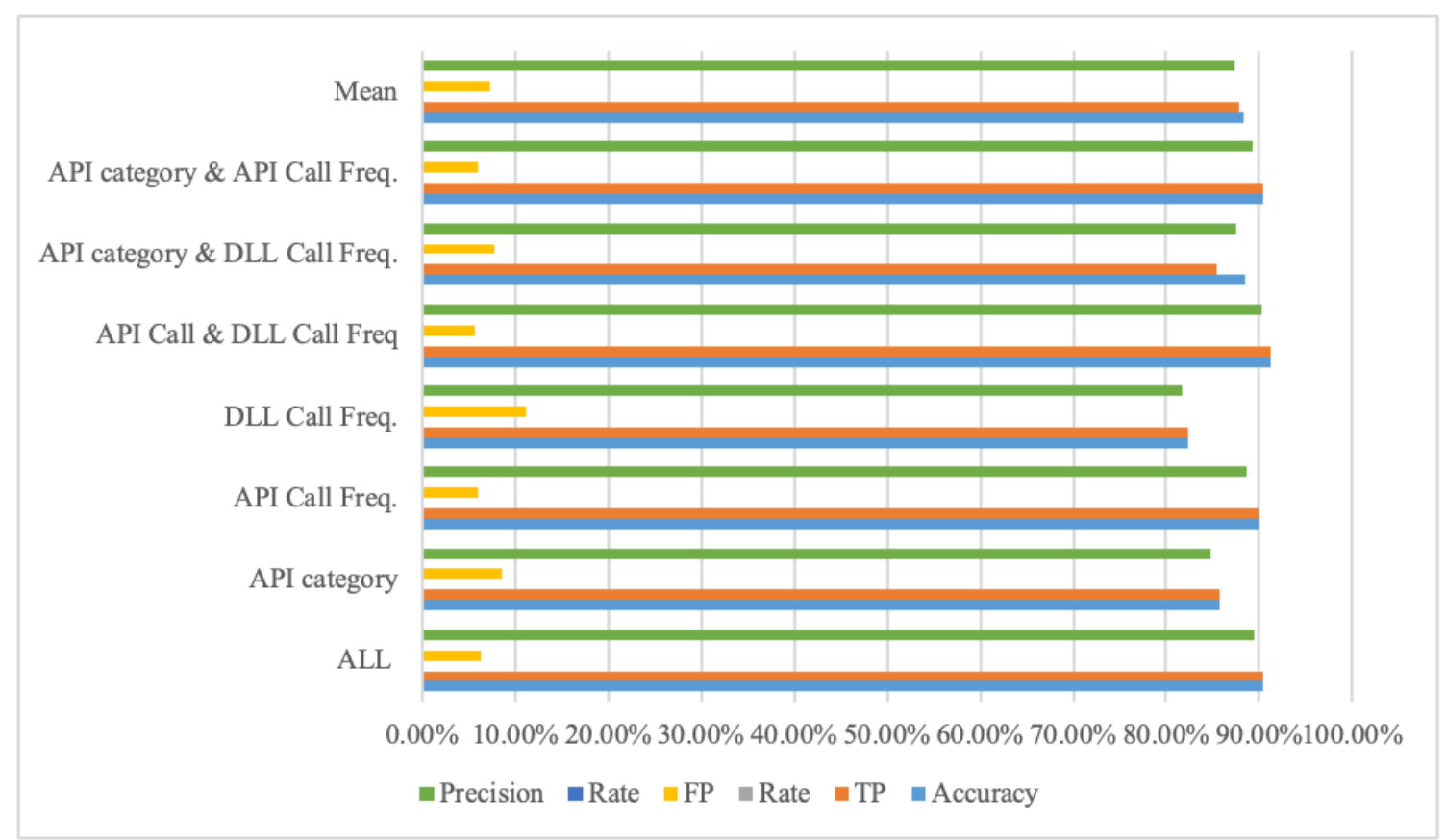

[Fig. 3] Results of Analysis when the Combinations of Features were Applied

In general, the results when only the DLL call frequency was applied as a feature showed the lowest performance. In addition, when the combination of API call frequency and DLL call frequency was applied to machine learning, the results showed the best performance. Most of cases where only one of the features was used showed the accuracy not exceeding $90 \%$, and the case where only the API call frequency was used showed the highest performance among the foregoing cases.

As a result, it is effective with an accuracy of more than $90 \%$ to determine the features of malware and judge malicious codes using APIs and DLLs. 


\section{Conclusion}

As malware has been diversified and studied, there are difficulties in detecting and analyzing the malware with existing systems, and it is very inefficient in terms of time and costs for analysts to firsthand analyze diverse kinds of malware. In this paper, features for classifying malware families were selected and the efficiency was analyzed through experiments using the available data. For the analysis, the data mining tools named Cuckoo Sandbox and WEKA, which are open source tools, were used. The performances when diverse combinations of the three features; API category frequency, API call frequency, and DLL call frequency, were checked. Based on the results, when only the DLL call frequency was applied, it showed relatively low performance. On the other hand, when only the API call frequency was applied, the results showed the best performance. Among the various combinations of the features, the combination of the API call frequency and DLL call frequency showed the highest performance. Through the foregoing, it can be seen that just combining several features does not show good performance, but efficient combinations of meaningful and relevant features show good performance and results.

In this paper, it was possible to confirm the accuracy of more than $90 \%$ in the detection classification using API and DLL targeting the actual malicious code, but it remains regrettable because a small number of 148 actual malicious codes were analyzed. In addition, there is a limitation in that it was not possible to detect more diverse malicious code families and select features because there are few malicious code samples. In future research, if the importance of collecting and classifying malicious code samples is increased and more diverse and accurate features are extracted, it will be more helpful for malicious code detection and classification.

When the proposed technique will be used as basis for future studies, other relevant analysis tools can be easily applied because open source tools were used, and other criteria can be set and used according to the analysis targets or the purposes of studies. As one method, it is possible to conduct a study on detection and classification through the same method for malware running on Linux systems other than the Windows system targeted in this paper.

Since not only diverse kinds of malware are found but also the variants of existing types of malware are found due to the characteristics of malware, it is expected that the content of this study will contribute to other malware analysis related studies.

\section{Acknowledgments}

This research was supported by the 2018 Yeungnam University Research Grant(218A061016, 218A380138) and the National Research Foundation of Korea(NRF) grant funded by the Korea government (MSIT) (No.2018R1D1A1B07050647).

\section{References}

[1] Lokesh Veeramacheneni, Information Security Risk Analysis Methids : AHP and Fuzzy Comprehensive Method, Asiapacific Journal of Convergent Research Interchange, (2015), Vol.1, No.2, pp.19-24.

[2] Hyun Chul Jung, Kwang-Kyu Seo, Prioritizing Cloud Service Threats for Succession to Information Security Management System, International Journal of Digital Contents and Applications for Smart Devices, (2020), Vol.7, No.1, pp.1-8.

[3] Manish Kumar Ahirwar, Piyush Kumar Shukla, Mnaish Sahu, and Sachin Goyal, Graph Theory \& Support Vector Machine based Mathematical Method for Malware Detection: A Ubiquitous Computing Based Approach, International 
Journal of Digital Contents and Applications for Smart Devices, (2015), Vol.2. No.2, pp.9-22.

[4] N.Thirupathi Rao, Debnath Bhattacharyya, Protocol, Security Issues and Challenges in Mobile Ad Hoc Networks: An Extensive Review, International Journal of Reliable Information and Assurance, (2020), Vol.8, No.1, pp.27-36.

[5] https://cuckoosandbox.org, Dec 15 (2020)

[6] https://www.cs.waikato.ac.nz/ml/weka/, Dec 15 (2020)

[7] S. S. Hansen, T. M. T. Larsen, M. Stevanovic, J. M. Pedersen, An approach for detection and family classification of malware based on behavioral analysis, 2016 International conference on computing, networking and communications, IEEE, (2016), pp.1-5, DOI Bookmark: 10.1109/ICCNC.2016.7440568

[8] I. K. Cho, E. G. Im, Malware Family Recommendation using Multiple Sequence Alignment, Journal of KIISE, (2016), Vol.43, No.3, pp.289-295, https://doi.org/10.5626/JOK.2016.43.3.289

[9] https://en.wikipedia.org/wiki/Multiple_sequence_alignment, Dec 15 (2020)

[10] M. Z. Rafique, P. Chen, C. Huygens, W. Joosen, Evolutionary Algorithms for Classification of Malware Families through Different Network Behaviors, Proceedings of the 2014 Annual Conference on Genetic and Evolutionary Computation, Association for Computing Machinery, pp.1167-1174, July 12 - 16, (2014), Vancouver BC Canada, https://doi.org/10.1145/2576768.2598238

[11] C. T. Lin, N. J. Wang, H. Xiao, C. Eckert, Feature Selection and Extraction for Malware Classification, Journal of Information Science and Engineering, (2015), Vol.31, No.3, pp.965-992. 